photographs or the movement of television cameras. He briefly described the bathyscaph, for the construction of which M. Jacques Piccard was largely responsible. The craft is similar in principle to the lighter-than-air balloons designed by M. Piccard's father. The buoyancy is obtained by using a float filled with petrol, and, by adjusting ballast and releasing small quantities of petrol, the trim of the craft may be alterod so that it will ascend or descend at the will of the operator. Below the float is a cabin, two metres in diameter, which will hold two men. Two conical windows of 'Perspex' permit observation from the cabin.

The depth of 1,100 metres is the greatest that is available in the neighbourhood of Capri, from where the bathyscaph is now operating. The cabin is designed to withstand a pressure equivalent to 6,000 metres of water, and as the petrol compartment of the float is open to the water, this is not affected by changes of pressure. There is thus no reason why the craft should not be able to descend to all but the deepost oceanic areas.

Dr. Dietz described in some detail the descent he had made. The dive lasted two and a half hours and included about forty-five minutes on the bottom. Some light was visible down to a depth of 525 metres.
At greater depths than this, floodlights were switched on intermittently. Some scattering particles, presumed to be zooplankton, were observed at all depths, but the maximum amount of life was found between 500 and 700 metres. The sea bottom appeared to be composed of soft brown mud, and there was little evidence of bottom life. Three small fish were seen near the bottom, however, and some mounds and burrows similar to those photographed by Dr. Laughton were observed. During the more rapid ascent, which took only forty minutes, a turbulent eddy of water travelling with the bathyscaph made observation difficult.

In the short discussion which followed the papers, Prof. T. G. Cowling asked Dr. Laughton whether the flat areas of sediment found on some parts of the ocean floor would cover a buried topography of the basement rocks. Dr. Laughton replied that this was almost cortainly the case.

Dr. B. J. Mason remarked that he was not surprised to learn that magnetic spherules of industrial origin were transported large distances from the areas where they were liberated into the atmosphere. In his work he had had experience of salt particles from the sea being carried several hundred miles inland.

R. D. Addas

\title{
POWER FROM NUCLEAR FUSION
}

\begin{abstract}
A SESSION of Section A (Physics) of the British Association was held in Dublin on September 6 under the prophetic title: "The Industrial Applications of Thermonuclear Reactions". This was under the chairmanship of Prof. T. G. Cowling, and followed his presidential address on "Stellar Energy and Stellar Evolution", which had included a discussion of thermonuclear reactions in the stars.

Two papers were read; the first, by J. D. Lawson (U.K. Atomic Energy Authority, Harwell), consisted of a brief review of the basic physics of the reaction process and the properties of the hot gas. The second, by Sir George Thomson, dealt more specifically with the problem of containing the hot gas out of contact with the relatively cool walls of its container. After the papers there was a brief but animated discussion.

Mr. Lawson said that if an efficient method could be found of burning to helium the deuterium which occurs in water then the world's fuel problem would be solved virtually for all time. Fusion reactions between ordinary light hydrogen nuclei (protons) are important in the stars, but would be almost undetectable in any conceivable terrestrial system; reactions employing the heavy hydrogen isotopes, deuterium and tritium, on the other hand, are about $10^{25}$ times more probable. A mixture of deuterium and tritium is by far the most 'inflammable' fuel ; unfortunately, however, tritium does not occur naturally. It can nevertheless be bred by capturing the neutrons emitted during fusion in the light isotope of lithium, which decays into tritium and helium. Lithium is not a common metal (reserves are probably comparable with those of uranium), and the ultimate aim must be to use a cycle based on deuterium. The rate of reaction for this cycle is about one hundred times less than that for the tritium/ deuterium cycle, and the problem of making a powerproducing system is considerably more difficult.
\end{abstract}

Temperatures of a few million degrees are required for fusion reactions to be detected, but for appreciable power to be produced at least a hundred million degrees is necessary. At such temperatures the gas is completely ionized. Radiation is in the form of bremsstrahlung (or 'free-free' electron transitions); its intensity increases as the square root of the temperature, and is proportional to the volume of the gas and the square of the density. This departure from the behaviour of a 'black body' is due to the fact that the range of the photons in the gas is very large compared with the linear dimensions of the system, so that the gas is almost transparent. Now the rate of reaction is also proportional to the volume and the square of the density, and hence the ratio of reaction power to radiated power is a function of temperature only. The two are equal at the 'critical temperature', which is about 150 million deg. for deuterium and 30 million deg. for a tritium/deuterium mixture.

It does not follow, however, that if a mass of fuel is heated to the critical temperature it can be 'set off', as in chemical explosion or combustion. The reason for this illustrates another difference between terres. trial and celestial systems; the ranges of the reaction products are very long, so that the neutrons are bound to escape from the gas without imparting their energy to it, though the charged reaction products can in principle be retained by means of suitable electric or magnetic fields.

The discussion so far illustrates some of the basic features of the reaction process, and indicates the difference in scale between a fusion reactor on one hand, where mean free paths are long compared with the linear dimensions of the system, and stars on the other hand, where the mean free paths are relatively short.

The central problem, however, is not that of producing a hot gas, difficult though this is, but of 
devising a system which produces more power than it consumes. A fission system needs no power to prime it, since, once a suitable lattice of fuel and moderator is assembled, heat appears. In a fusion system, on the other hand, electrical energy is required to heat the gas and to provide external magnetic fields. It is no use, for example, making a system which consumes one megawatt of electricity and only produces a further megawatt of heat, since some three megawatts of heat are required in the first place to produce one megawatt of electricity. Conditions for a favourable power balance are very stringent indeed; the gas must be heated above the critical temperature in almost complete isolation from the walls for long enough for at least a few per cent of the nuclei to be burnt.

Mr. Lawson ended by saying that the problems still to be solved appear at the moment to be formidable, and that it is not yet clear which road, if any, will lead to success.

Sir George Thomson then spoke on the problem of containing the hot gas. He started by saying that it takes a great deal of energy to heat the gas to the required temperature of $10^{8}-10^{8} \mathrm{deg}$., and that this heat must not be allowed to escape until a substantial fraction of the gas has been burnt. For the densities likely to be used, say, $10^{16}$ particles $/ \mathrm{cm} .^{3}$, deuterium must be contained for about a second if a net power yield is to be obtained.

A gas in the completely ionized state which occurs at these temperatures is known as a 'plasma'; the mean free paths of the particles are very great, and the electrical conductivity may exceed that of copper at room temperature. In consequence of this high mean free path one cannot hope to keep the gas together by the entanglement with each other of the particle motions; an attempt to use this method would result either in a bomb, or in a system of quite impractical size.

Now heat can be lost by radiation, conduction and convection. Radiation losses are serious, but acceptable; this is fortunate since there seems to be no way of preventing them. Loss by conduction, on the other hand, would be prohibitive if nothing were done to prevent it. Sir George then did a simple calculation to show that, for a density of $10^{16}$ particles $/ \mathrm{cm} .^{3}$ and a temperature of $10^{8} \mathrm{deg}$., loss of heat by conduction from a sphere would exceed that by radiation unless the radius were greater than $100 \mathrm{~km}$.

At first sight, one might hope to gain by departing from a Maxwell-Boltzmann gas. This is not possible, however, since the scattering cross-section greatly exceeds that for the nuclear reaction in deuterium, and consequently the ions will achieve a fair approximation to equilibrium if the high temperature is sustained long enough for a substantial fraction of them to react. In these circumstances Boltzmann's relation between potential energy and density will hold roughly, and this shows that if the density at the edge is to be really small compared with that at the centre there must be a potential difference between these which is at least a substantial multiple of $k T / e$, where $k$ is Boltzmann's constant.

This shows that containment is ultimately by electric fields, though this fact may be well concealed. Containment by electrostatic fields applied externally is indeed impossible, but large internal electric fields can arise from a very small separation of positive and negative charges. A field so directed as to stop positive ions from striking the walls will impel electrons to do so; this must be stopped, and it is hoped to achieve this by anchoring the electrons by means of a magnetic field. Electron orbits in the presence of a magnetic field are 'coiled up' and the rate of diffusion is greatly reduced. If, however, an electric field is also present the particles move in trochoidal orbits, in an average direction perpendicular to both the electric and magnetic fields. Scattering does indeed result in a gradual shift of the orbits, which are pushed downhill, so to speak, by the electric field, but when the magnetic field is strong this process is very slow. The steady state predicted by the Boltzmann expression will not in practice even be approached by the electrons.

A full analysis of the behaviour of the plasma under the action of both electric and magnetic fields would be very complicated. However, in the special case of the steady state where the conductivity of the plasma is high, the relatively simple result is obtained that a current at right angles to the magnetic field will cause a gradient of pressure perpendicular to both and equal (in suitable units) to their product. The current is roughly what it would be if there were no magnetic field. It remains to adjust this gradient of pressure so as to keep the gas away from the walls of the vessel. One way of doing this is to use the 'pinch effect', which occurs when a heavy current is passed through a gas contained in a cylindrical vessel. The current elements attract one another, and a narrow current-channel forms away from the walls of the cylinder. Another way of looking at this is to regard the lines of force due to the current, which form rings round it, as possessing a tension equal to $H^{2} / 8 \pi$ (where $H$ is the magnetic field).

The drawback of this arrangement is that the current-channel is unstable. If for any reason it bends, the magnetic lines become crowded together inside the bend ; this increases the magnetic pressure there and the bend is accentuated. It is not easy to avoid this, which may be an outstanding difficulty.

Another method is to use a magnetic field produced externally which might, for example, be arranged to go tyrewise round a torus. The setting up of such a field will produce currents in the gas, this time round the cross-section of the torus. Such currents have a magnetic field which tends to neutralize the applied field inside them, and the gas at the centre is held in by the sideways pressure of the tyrewise lines of force, which is still strong in the outer parts of the cross-section. Here again instabilities are troublesome, although it seems that a combination of the two sets of currents may have possibilities.

The third form of heat-loss, by convection, is essentially associated with turbulent motion, and does not occur in a steady state such as one might hope to obtain in a heated plasma. Where instabilities occur, however, as in a pinched discharge, one would expect large losses of a convective character due to masses of gas being thrown against the walls. This is one reason why instabilities are undesirable.

Finally, how can energy be extracted from the hot gas? The heat transferred through the walls of the containing vessel could be used for raising steam, but a more attractive possibility is to make use of the magnetic properties of the plasma to induce electrical power directly in some external coupled circuit. It is not yet clear how this could be done, but the possibility exists. 
Sir George ended by saying that he had sufficient confidence in the ingenuity of electrical engineers to believe that, since no fundamental reason had been found in ten years which made a fusion reactor impossible, this amounted to proof that it could be made.

Among the topics raised in the discussion after the talks was the possibility of using thermonuclear neutrons for breeding fissile material, and the point was made that this might be worth while even if the thermonuclear reaction cycle itself was not selfsustaining. It is, however, too early yet to say how this would compete with 'conventional' breeder systems.

Asked whether power might be achieved using the 'cold fusion' process, whereby the hydrogen and deuterium nuclei in a mesic molecule coalesce and fling out the meson with high energy, Mr. Lawson said that it was unlikely that a meson, suffioiently long lived to make such a process economical, existed. It was agreed, however, that this was a very interesting phenomenon, and showed that quite unexpected things may still happen.

The discussion was not entirely satisfactory, however, since some of the most interesting questions could not be answered for reasons of security. There is no doubt that Prof. Cowling echoed the feelings of the meeting when he said, in thanking the speakers, that he looked forward to the day when it would not be necessary to answer questions by referring only to the latest published information.

J. D. LAwSON

\section{THE DEFENCE RESEARCH BOARD, CANADA}

\section{By C. A. POPE}

$\mathrm{T}$ HE Defence Research Board, Canada's largest scientific organization, celebrated its tenth anniversary recently.

During the later stages of the Second World War, the efforts of Canadian scientists indicated that research could provide vitally important support to the Armed Forces; and the desire of the National Research Council and other scientists involved to return to civilian research pursuits made obvious the creation of a permanent defence research organization. Hence was born the Defence Research Board on April 1, 1947. The first chairman was Dr. Omond M. Solandt, a youthful, war-time operational research scientist, who was succeeded in March 1956 by Dr. A. Hartloy Zimmerman, previously a member of the Defence Resoarch Board and vice-chairman of the organization for about a year.

The Board is believed to be unique as a scientific body because it is directed by civilians although integrated within the Department of National Defence, and it has surmounted successfully a series of growing pains and has achieved international recognition for its efforts on behalf of defence research.

Among the problems overcome during the first ten years of the Board's existence were the initial phase of organization, the establishment of a carefully planned scientific programme-interrupted by urgent Korean War requirements-and a widespread re-organization following the end of hostilities with emphasis on now and increasingly important military fields.

The keystone of defence science in Canada is the Defence Research Board itself, which consists at present of fourteen members, six of whom are ex-officio and six of whom represent Canadian science both from the universities and from industry. The chairman and vice-chairman are permanent, salaried members of the Board. The chairman is appointed by the Governor-General-in-Council; his status is that of a chief of staff and, as such, he sits on the Chiefs of Staff Committee. He possessos many of the financial and other powers of a deputy minister, since he reports directly to the Minister of National Defence.
The Board operates with three main objectives in mind : (1) To use existing Canadian research and development facilities wherever possible to meet the neods of the Armed Forces. (2) To maintain collaboration with the United Kingdom and United States on a partnership basis. (This permits the Board to concentrate its efforts on a limited number of problems of particular importance to Canada or for which Canada possesses unique resources or facilitios.) (3) To promote in every way possible the progress of standardization, a subject of vital Canadian concern.

The value of the Defence Research Board's physical assets, comprising mainly eleven well-equipped pormanent scientific laboratories, totals about $32,000,000$ dollars. Almost 4,500,000 dollars was invested in the facilities which existed at Valcartier, P.Q., and Suffield, Alta., when they were Armed Services' establishments prior to 1947 . Now construction during the past decade, therefore, at these and at the Board's other establishments represents a total expenditure of about $27,500,000$ dollars. The resulting modern research facilities will help to serve for many years to come the defence scientific interests of Canada.

The total appropriation requested for defence research and development in 1956-57 was almost $80,000,000$ dollars, of which approximately $24,000,000$ dollars will be used for research. This sum covers the operational costs of the Board's establishments and its liaison offices in London and Washington, and its annual 1,000,000 dollars for its programme of grants and contracts for fundamental research with Canadian universities. The remainder of the appropriation covers the development activities of the three Services.

The staff of the Defence Research Board has increased from a handful of scientists, seconded Service officers and administrative personnel early in 1947 to a total of 2,800 , of whom 650 are employed in professional categories. In addition, scientists and others with highly specialized knowledge from the scientific community sit voluntarily in consulting capacities on a wide variety of panels and committees concerned with the Board's programme. 